\title{
Muon capture rates: Evaluation within the quasiparticle random phase approximation
}

\author{
Fedor Šimkovic $\odot,{ }^{1,2,3, *}$ Rastislav Dvornický $\odot,{ }^{1,4, \dagger}$ and Petr Vogel $\oplus^{5, *}$ \\ ${ }^{1}$ Faculty of Mathematics, Physics and Informatics, Comenius University in Bratislava, 84248 Bratislava, Slovakia \\ ${ }^{2}$ Bogoliubov Laboratory of Theoretical Physics, Joint Institute for Nuclear Research, 141980 Dubna, Russia \\ ${ }^{3}$ Institute of Experimental and Applied Physics, Czech Technical University in Prague, 11000 Prague, Czech Republic \\ ${ }^{4}$ Dzhelepov Laboratory of Nuclear Problems, Joint Institute for Nuclear Research, 141980 Dubna, Russia \\ ${ }^{5}$ Kellogg Radiation Laboratory and Physics Department, Caltech, Pasadena, California 91125, USA
}

(Received 5 June 2020; revised 22 July 2020; accepted 17 August 2020; published 1 September 2020)

\begin{abstract}
The quasiparticle random phase approximation is used in evaluation of the total muon capture rates for final nuclei participating in double- $\beta$ decay. Several variants of the method are used, depending on the size of the single-particle model space used, or treatment of the initial bound muon wave function. The resulting capture rates are all reasonably close to each other. In particular, the variant that appears to be most realistic results in rates that are in good agreement with the experimental values. There is no necessity for an empirical quenching of the axial current coupling constant $g_{A}$. Its standard value $g_{A}=1.27$ seems to be adequate.
\end{abstract}

DOI: 10.1103/PhysRevC.102.034301

\section{INTRODUCTION}

The capture of negative muons from the $1 s$ muonic atom orbit,

$$
\mu^{-}+(Z, N) \rightarrow v_{\mu}+(Z-1, N+1)^{*},
$$

has been studied in detail for a long time (see the classic reviews by Walecka [1], Mukhopadhyay [2], and Measday [3]). Experimental determination of the total muon capture rate is relatively straightforward, therefore it is known for many stable elements, sometimes even for the separated isotopes $[4,5]$.

The nuclear response in this semileptonic weak process is governed by the momentum transfer of the order of muon mass. The region of the excited nuclear states near the giant dipole resonance dominates in the final nuclei since the phase space as well as nuclear response give preference to low excitation energies. These features lead to the recent revival of interest in the muon capture as a testing ground for theoretical description of weak nuclear processes. In particular, the question of the so-called axial current quenching phenomenon is widely discussed in connection with the evaluation of $0 v \beta \beta$ decay nuclear matrix elements.

It is well known that using the nuclear shell model leads to the prediction of the allowed Gamow-Teller $\beta$ decays, as well as of the two-neutrino double- $\beta$ decays $(2 v \beta \beta)$, which are too fast compared to the experimental lifetimes. The corresponding enhancement factors are approximately the same for all nuclei in the same shell, thus they can be conveniently

\footnotetext{
*simkovic@teller.dnp.fmph.uniba.sk

†rastonator@gmail.com

‡pvogel@caltech.edu
}

described by a phenomenological effective axial vector coupling constant $g_{A}^{\text {eff }}$ that is smaller than the $g_{A}=1.27$ deduced from the free neutron $\beta$ decay [6,7]. Since the $2 \nu \beta \beta$ decay is nothing else than two GT transitions occurring at once, similar quenching appears when the $2 v \beta \beta$ rate is calculated in the shell model [8]. Recent careful analysis [9] suggests that when all nuclear correlations, including the effects of the two-body weak currents, and a proper treatment of effective operators, are included, the GT transition strength is correctly described without the need to use the quenching idea. However, the advanced treatment of nuclear correlations, as in Ref. [9], is not yet available for evaluation of the rate of the $0 \nu \beta \beta$ and $2 v \beta \beta$ decays, or of the muon capture.

The magnitude of quenching, i.e., the amount $q \leqslant 1$ of the ratio $q=g_{A}^{\text {eff }} / g_{A}$, is nuclear model dependent. For example, when the interaction boson model version IBM-2 is used [10], the corresponding $q$ is considerably smaller than in the case of the shell-model treatment [8]. Within the quasiparticle random phase approximation (QRPA) the situation is more complex, since the quenching amount $q$ is strongly correlated with the particle-particle effective coupling parameter $g_{p p}$, which is often adjusted to correctly describe the $2 \nu \beta \beta$ decay half-life $[11,12]$.

The quenching phenomenon has been firmly established for the low momentum transfer GT-type nuclear transitions, governed by the selection rules $\Delta I \leqslant 1, \Delta \pi=0$ and involving dominantly the $\sigma \tau$ operator. However, the neutrinoless double- $\beta$ decay $(0 v \beta \beta)$ involves momentum transfer $q \approx 100$ $\mathrm{MeV}$, with no restriction on angular momentum and parity change. This makes the muon capture, with analogous unrestrictive selection rules and a magnitude of the momentum transfer, an attractive testing ground for nuclear model description of the nuclear matrix elements for the $0 v \beta \beta$ decay. One of the examples of the recent effort along these lines is in Ref. [13] dedicated mostly to the experimental study of the 
nuclear $\gamma$ radiation following muon capture in the $\left(\mu^{-}, v x n\right)$ reactions on the final nuclei involved in $\beta \beta$ decay.

In this work we use the QRPA to evaluate the total muon capture rates for the $0 \nu \beta \beta$ decay candidate nuclei and compare them with the experiment. The excitation energy and multipolarity distributions are also presented. Previous analogous calculations of the total muon capture rate lead to ambiguous conclusions. References [14-16] use a version of QRPA and conclude that none, or only mild quenching is needed. Similarly, the shell model applied to the muon capture on ${ }^{16} \mathrm{O}$ in Ref. [17] also required only minimal quenching $g_{A}^{\text {eff }} / g_{A} \approx 0.95$. On the other hand, in Ref. [18] based on QRPA quite strong quenching $g_{A}^{\text {eff }} / g_{A} \approx 0.5$ is required to describe the total muon capture rates of the $0 \nu \beta \beta$-decay candidate nuclei.

This motivates us to use the formalism closely related to the one used previously for the evaluation of the $0 v \beta \beta$ nuclear matrix elements [12]. The paper is organized as follows: In Sec. II the formalism is briefly described. In Sec. III we discuss the choice of input parameters and study the corresponding uncertainties. In Sec. IV the results are shown and conclusion about the amount of needed $g_{A}$ quenching is discussed. Our conclusions are presented in Sec. V.

\section{FORMALISM}

\section{A. Effective weak Hamiltonian and $T$ matrix}

The effective weak lepton-nucleus interaction Hamiltonian is of the standard form

$$
H_{\mathrm{w}}(x)=\frac{G_{\beta}}{\sqrt{2}} \bar{v}_{\mu}(x) \gamma_{\alpha}\left(1-\gamma_{5}\right) \mu(x) J_{L}^{\alpha}(x)+\text { H.c. }
$$

Here $G_{\beta}=G_{F} \cos \theta_{C}, \theta_{C}$ is the Cabbibo angle. $\mu(x)$ and $v_{\mu}(x)$ are the muon and muonic neutrino fields, respectively. $J_{L}^{\alpha}(x)$ is the $V-A$ hadronic current at the nucleon level renormalized by the strong and electromagnetic interactions. We have

$$
\begin{aligned}
J_{L}^{\alpha}= & \left\langle n\left(p^{\prime}\right)\left|\bar{d} \gamma^{\alpha}\left(1-\gamma_{5}\right) u\right| p(p)\right\rangle \\
= & \bar{n}\left(p^{\prime}\right)\left[g_{V}\left(q^{2}\right) \gamma^{\alpha}+i g_{M}\left(q^{2}\right) \frac{\sigma^{\alpha \beta}}{2 m_{p}} q_{\beta}\right. \\
& \left.-g_{A}\left(q^{2}\right) \gamma^{\alpha} \gamma_{5}-g_{P}\left(q^{2}\right) q^{\alpha} \gamma_{5}\right] p(p),
\end{aligned}
$$

where $m_{p}$ is the nucleon mass, $q_{\mu}=\left(p^{\prime}-p\right)_{\mu}$ is the momentum transfer, and $p^{\prime}$ and $p$ are the four momenta of neutron and proton, respectively. For the nucleon form factors $g_{V}\left(q^{2}\right), g_{M}\left(q^{2}\right), g_{A}\left(q^{2}\right)$, and $g_{P}\left(q^{2}\right)$ we use the usual dipole parametrization

$$
\begin{aligned}
\frac{g_{V, M}\left(q^{2}\right)}{g_{V, M}} & =\left(1-\frac{q^{2}}{M_{V}^{2}}\right)^{-2}, \\
\frac{g_{A}\left(q^{2}\right)}{g_{A}} & =\left(1-\frac{q^{2}}{M_{A}^{2}}\right)^{-2},
\end{aligned}
$$

with $M_{V}=850 \mathrm{MeV}$ and $M_{V}=1086 \mathrm{MeV}$ and $g_{V} \equiv g_{V}(0)=1, \quad g_{A} \equiv g_{A}(0)=1.269, \quad g_{M} \equiv g_{M}(0)=$ $\left(\mu_{p}-\mu_{n}\right) g_{V}=3.70$. The induced pseudoscalar form factor is given by the PCAC relation

$$
g_{P}\left(q^{2}\right)=\frac{2 m_{p}}{m_{\pi}^{2}-q^{2}} g_{A}\left(q^{2}\right),
$$

where $m_{\pi}$ is the pion mass.

Next, it is necessary to reduce the nucleon current to the nonrelativistic form. By keeping terms up to $1 / m_{p}$ and neglecting terms $O\left(q_{0}^{2} / m_{p}^{2}\right)$ we get [19]

$$
\begin{aligned}
J_{L}^{0}= & g_{V}\left(q^{2}\right)-g_{A}\left(q^{2}\right) \frac{\boldsymbol{\sigma} \cdot\left(\mathbf{p}+\mathbf{p}^{\prime}\right)}{2 m_{p}}+g_{P}\left(q^{2}\right) \frac{q_{0} \boldsymbol{\sigma} \cdot \mathbf{q}}{2 m_{p}}, \\
\mathbf{J}_{L}= & -g_{A}\left(q^{2}\right) \boldsymbol{\sigma}+g_{P}\left(q^{2}\right) \frac{\mathbf{q} \boldsymbol{\sigma} \cdot \mathbf{q}}{2 m_{p}}+g_{V}\left(q^{2}\right) \frac{\mathbf{p}+\mathbf{p}^{\prime}}{2 m_{p}} \\
& +\left(g_{V}\left(q^{2}\right)+g_{M}\left(q^{2}\right)\right) i \frac{\boldsymbol{\sigma} \times \mathbf{q}}{2 m_{p}} .
\end{aligned}
$$

Note that usually nonrelativistic reduction is performed in the Breit frame $\left(q_{0}=0\right.$ and $\mathbf{p}+\mathbf{p}^{\prime}=0$ [19]), e.g., in the case of the $0 v \beta \beta$ decay [12] and the elastic electron nucleon (nucleus) scattering of neutrinos on nuclei, etc. In these processes the energies of incoming and outgoing leptons are approximately the same or negligible.

Unlike that, the calculation of muon capture is performed in the proton rest frame where $q_{0}=E_{\mu}-E_{v}, \mathbf{q}=\mathbf{p}^{\prime}-\mathbf{p}=$ $-\mathbf{p}_{v}$, and $\mathbf{p}^{\prime}+\mathbf{p}=-\mathbf{p}_{v}[20,21]$, since $p=0$ in this frame. $E_{\mu}=m_{\mu}-\varepsilon_{b}$ is the energy of the bound muon in the $\kappa=-1$ state in the muonic atom, where $\varepsilon_{b}$ is the binding energy. $E_{v}$ and $\mathbf{p}_{v}$ are energy and momentum of emitted neutrino, respectively. $p_{v}=\left|\mathbf{p}_{v}\right|=E_{v}$ since we neglect neutrino mass. Thus, within the nonrelativistic impulse approximation, the hadronic current for muon capture on nuclei is expressed as

$$
\begin{aligned}
J_{L}^{0}= & g_{V}\left(q^{2}\right)+g_{A}\left(q^{2}\right) \frac{\boldsymbol{\sigma} \cdot \mathbf{p}_{v}}{2 m_{p}}-g_{P}\left(q^{2}\right) \frac{q_{0} \boldsymbol{\sigma} \cdot \mathbf{p}_{v}}{2 m_{p}}, \\
\mathbf{J}_{L}= & -g_{A}\left(q^{2}\right) \boldsymbol{\sigma}+g_{P}\left(q^{2}\right) \frac{\mathbf{p}_{v} \boldsymbol{\sigma} \cdot \mathbf{p}_{v}}{2 m_{p}}-g_{V}\left(q^{2}\right) \frac{\mathbf{p}_{v}}{2 m_{p}} \\
& -i\left(g_{V}\left(q^{2}\right)+g_{M}\left(q^{2}\right)\right) \frac{\boldsymbol{\sigma} \times \mathbf{p}_{v}}{2 m_{p}} .
\end{aligned}
$$

Apart from a few small terms the structure of the current is the same as in the case of the $0 v \beta \beta$ decay.

The muon capture on nuclei occurs in the first order in weak interaction. The corresponding $S$ matrix is

$$
\left\langle f\left|S^{(1)}\right| i\right\rangle=2 \pi \delta\left(E_{f}+E_{v}-E_{i}-E_{\mu}\right)\left\langle f\left|T^{(1)}\right| i\right\rangle,
$$

where the $T$ matrix is

$$
\begin{aligned}
\left\langle f\left|T^{(1)}\right| i\right\rangle= & (-i) \frac{G_{\beta}}{\sqrt{2}} \int\left\langle f\left|J_{L}^{\alpha}(0, \mathbf{r})\right| i\right\rangle \\
& \times \Phi\left(E_{\mu}, \mathbf{r}\right) \gamma_{\alpha}\left(1-\gamma_{5}\right) \Phi\left(E_{\nu}, \mathbf{r}\right) d \mathbf{r} .
\end{aligned}
$$

Nuclear current takes the form

$$
J_{L}^{\alpha}(0, \mathbf{r})=\sum_{n=1}^{A} \tau_{n}^{-}\left(g^{\alpha 0} J_{L}^{0}+g^{\alpha k}\left(\mathbf{J}_{L}\right)^{k}\right) \delta\left(\mathbf{r}-\mathbf{r}_{n}\right)
$$


and wave functions of the bound $\kappa=-1$ muon $\Phi_{\mu}\left(E_{\mu}, \mathbf{r}\right)$ and emitted neutrino $\Phi_{\nu}\left(E_{\nu}, \mathbf{r}\right)$ are given by

$$
\begin{aligned}
& \Phi_{\mu}\left(E_{\mu}, \mathbf{r}\right)=\frac{1}{\sqrt{4 \pi}}\left(\begin{array}{c}
g_{-1}(r) \chi_{m} \\
-i f_{-1}(r)(\boldsymbol{\sigma} \cdot \hat{\mathbf{r}}) \chi_{m}
\end{array}\right), \\
& \Phi_{\nu}\left(E_{v}, \mathbf{r}\right)=\frac{1}{\sqrt{2}}\left(\begin{array}{c}
\chi_{m} \\
\frac{\sigma \cdot \hat{\mathbf{p}}_{v}}{E_{v}} \chi_{m}
\end{array}\right) e^{-i \mathbf{p}_{v} \cdot \mathbf{r}} .
\end{aligned}
$$

The energy of emitted neutrino follows from the energy conservation guaranteed by the $\delta$ function and is determined by the equation

$$
E_{v}+\sqrt{M_{f}^{2}+p_{v}^{2}}-\left(m_{\mu}-\varepsilon_{b}+M_{i}\right)=0 .
$$

Here, the energies of the initial $|i\rangle$ and final $|f\rangle$ states are $E_{i}=$ $M_{i}$ and $E_{f}=\sqrt{M_{f}^{2}+p_{v}^{2}}$, respectively. For medium-heavy nuclei the nuclear recoil energy $p_{v}^{2} /\left(2 M_{f}\right)$ is of the order of tens of $\mathrm{keV}$ and can be safely neglected, as well as the effect of center of mass of muon-nuclear system.

\section{B. Muon capture rate}

The differential muon capture rate summed over all final excited states $|\mathrm{k}\rangle$ can be written as

$$
d \Gamma=2 \pi \sum_{k} \delta\left(E_{v}+E_{k}-E_{i}-E_{\mu}\right) \sum_{\text {spin }}|\langle k|T| i\rangle|^{2} \frac{d \mathbf{k}}{(2 \pi)^{3}} .
$$

Here, the squared $T$ matrix is summed over all spin orientations of the neutrino and daughter nucleus and averaged over all spin orientations of the muon and the parent nucleus.

Inserting Eqs. (9) and (11) for the squared $T$-matrix element, we find the total capture rate. When only parity even operators, relevant for the ground-state expectation value, are kept, the total capture rate takes the form

$$
\Gamma=m_{\mu} \frac{\left(G_{\beta} m_{\mu}^{2}\right)^{2}}{2 \pi}\left(C_{V} B_{\Phi V}+C_{A} B_{\phi A}+C_{P} B_{\phi P}\right) .
$$

The quantities $B_{\Phi K}(K=V, A, P)$ are

$$
B_{\Phi K}=\sum_{k} \frac{E_{v_{k}}^{2}}{m_{\mu}^{2}} B_{\Phi K}^{k}\left(p_{v_{k}}\right),
$$

where $E_{v_{k}}=p_{v_{k}}=E_{\mu}+E_{i}-E_{k}$. The sum is over all states $|k\rangle$ in the nucleus $(Z-1, N+1)$ that can be reached by the corresponding operators involved in the squared matrix elements

$$
\begin{aligned}
B_{\Phi K}^{k}\left(p_{v_{k}}\right)= & \frac{1}{\hat{J}_{i}} \sum_{M_{i} M_{k}} \int \frac{d \Omega_{v}}{4 \pi} \\
& \times\left|\left\langle J_{k} M_{k}\left|\sum_{j=1}^{A} \tau_{j}^{-} e^{i \mathbf{p}_{v_{k}} \cdot \mathbf{r}_{i}} O_{K} \frac{\Phi_{g}\left(r_{i}\right)}{m_{\mu}^{3 / 2}}\right| J_{i} M_{i}\right\rangle\right|^{2} .
\end{aligned}
$$

Here, $\left|J_{i} M_{i}\right\rangle\left(\left|J_{k} M_{k}\right\rangle\right)$ is the initial (final) nuclear state with spin $J_{i}\left(J_{k}\right)$ and spin-projection $M_{i}\left(M_{k}\right), \hat{J}_{i}=2 J_{i}+1$, $\Phi_{g}(r)=g_{-1}(r) / \sqrt{4 \pi}$ and

$$
O_{V}=1, \quad O_{A}=\sigma_{j}, \quad O_{P}=\sigma_{j} \cdot \hat{\mathbf{p}}_{v_{k}} .
$$

The effective coupling constants in Eq. (14) are

$$
\begin{aligned}
C_{V}= & g_{V}^{2}\left(q^{2}\right)\left(1+\frac{p_{v}^{2}}{\left(2 m_{p}\right)^{2}}\right) \\
C_{A}= & g_{A}^{2}\left(q^{2}\right)+\left(g_{V}\left(q^{2}\right)+g_{M}\left(q^{2}\right)\right)^{2} \frac{p_{v}^{2}}{\left(2 m_{p}\right)^{2}} \\
C_{P}= & \frac{p_{v}^{2}}{\left(2 m_{p}\right)^{2}}\left(g_{A}^{2}\left(q^{2}\right)-2 g_{A}\left(q^{2}\right) g_{P}^{\mu}\left(q^{2}\right) \frac{2 m_{p}}{m_{\mu}}\right. \\
& \left.+\left(g_{P}^{\mu}\right)^{2}\left(q^{2}\right) \frac{p_{v}^{2}}{m_{\mu}^{2}}-\left(g_{V}\left(q^{2}\right)+g_{M}\left(q^{2}\right)\right)^{2}\right) .
\end{aligned}
$$

Here, the dimensionless pseudoscalar form factor is $g_{P}^{\mu}\left(q^{2}\right)=m_{\mu} g_{P}\left(q^{2}\right)=\frac{2 m_{p} m_{\mu}}{m_{\pi}^{2}-q^{2}} g_{A}\left(q^{2}\right)$. The coefficients $C_{V, A, P}$ only weakly depend on the neutrino momentum $p_{v_{k}}$. For a sake of simplicity they are not included in the calculation of $B_{\Phi K}$ but are evaluated for some average neutrino momentum $p_{v}$.

Often used [23-26] alternative reduction of the nucleon current to its nonrelativistic form is based on a renormalization procedure of nucleon current due to strong interaction of Ref. [22]. This, so-called Fujii-Primakoff form, of the constants $C_{V}, C_{A}$, and $C_{P}$ in Eq. (14) is

$$
C_{V}=G_{V}^{2}, \quad C_{A}=G_{A}^{2}, \quad C_{P}=G_{P}^{2}-2 G_{A} G_{P},
$$

with

$$
\begin{aligned}
& G_{V}=g_{V}\left(q^{2}\right)\left(1+\frac{p_{v}}{2 m_{p}}\right), \\
& G_{A}=-g_{A}\left(q^{2}\right)-\left(g_{V}\left(q^{2}\right)+g_{M}\left(q^{2}\right)\right) \frac{p_{v}}{2 m_{p}}, \\
& G_{P}=\left(g_{P}^{\mu}\left(q^{2}\right)+g_{A}\left(q^{2}\right)-g_{V}\left(q^{2}\right)+g_{M}\left(q^{2}\right)\right) \frac{p_{v}}{2 m_{p}} .
\end{aligned}
$$

These two forms of the constants $C_{V}, C_{A}$, and $C_{P}$ differ in the recoil order terms $p_{v} /\left(2 m_{p}\right)$.

In order to make the analogy to the evaluation of the $0 \nu \beta \beta$ matrix element more explicit we introduce Fermi, GamowTeller, and tensor squared matrix elements,

$$
B_{\Phi F}=B_{\Phi V}, \quad B_{\Phi G T}=B_{\Phi A}, \quad B_{\Phi T}=3 B_{\Phi P}-B_{\Phi A},
$$

Their explicit form will be given in Sec. IIF. The muon capture rate then takes the form

$$
\begin{aligned}
\Gamma & =m_{\mu} \frac{\left(G_{\beta} m_{\mu}^{2}\right)^{2}}{2 \pi} \\
& \times\left(g_{A}^{\text {eff }}\right)^{2}\left(C_{F} \frac{B_{\Phi F}}{\left(g_{A}^{\text {eff }}\right)^{2}}+C_{G T} B_{\Phi G T}+C_{T} B_{\Phi T}\right),
\end{aligned}
$$

where the $\left(g_{A}^{\text {eff }}\right)^{2}$ appears as a scale parameter. The constants $C_{F}, C_{T}$, and $C_{G T}$ are given by

$$
\begin{aligned}
C_{F} & =G_{V}^{2}, \quad C_{T}=\frac{1}{\left(g_{A}^{\text {eff }}\right)^{2}} \frac{C_{P}}{3}, \\
C_{G T} & =\frac{1}{\left(g_{A}^{\text {eff }}\right)^{2}}\left(C_{A}+\frac{C_{P}}{3}\right) .
\end{aligned}
$$


TABLE I. The coefficients $C_{F}, C_{G T}$, and $C_{T}$ [see Eq. (23)] calculated within the present approach [see Eq. (18)] and in the Fujii-Primakoff approximation [see Eq. (19)].

\begin{tabular}{lcccccccc}
\hline \hline$E_{v}$ & & \multicolumn{3}{c}{ present approach } & & \multicolumn{3}{c}{ Fujii-Primakoff } \\
\cline { 3 - 5 } \cline { 7 - 9 }$(\mathrm{MeV})$ & $g_{A}^{\text {eff }}$ & $C_{F}$ & $C_{G T}$ & $C_{T}$ & & $C_{F}$ & $C_{G T}$ & $C_{T}$ \\
\hline 75 & 0.80 & 0.976 & 0.797 & -0.241 & & 1.054 & 1.165 & -0.333 \\
& 1.00 & 0.976 & 0.821 & -0.197 & & 1.054 & 1.091 & -0.296 \\
& 1.27 & 0.976 & 0.847 & -0.158 & & 1.054 & 1.030 & -0.265 \\
85 & 0.80 & 0.965 & 0.805 & -0.239 & & 1.052 & 1.203 & -0.359 \\
& 1.00 & 0.965 & 0.823 & -0.197 & & 1.052 & 1.117 & -0.317 \\
& 1.27 & 0.965 & 0.844 & -0.159 & & 1.052 & 1.048 & -0.282 \\
95 & 0.80 & 0.955 & 0.818 & -0.234 & & 1.051 & 1.241 & -0.385 \\
& 1.00 & 0.955 & 0.828 & -0.195 & & 1.051 & 1.145 & -0.337 \\
& 1.27 & 0.955 & 0.844 & -0.159 & & 1.051 & 1.067 & -0.298 \\
\hline \hline
\end{tabular}

In Table I we compare the $C_{F}, C_{G T}$, and $C_{T}$ coefficients introduced in the present approach with those governing the Fujii-Primakoff approach. We note that the coefficients $C_{K}$ $(K=F, G T, T)$ are less dependent on the neutrino energy $E_{v}$ than the coefficients $C_{V}, C_{A}$, and $C_{P}$, and only slightly dependent on the parameter $g_{A}^{\text {eff }}$.

In this paper we use two alternative ways to include the bound muon wave function in the muon capture rate formula. Traditionally, in order to simplify the calculation, it is assumed that the muon wave function and nuclear matrix elements can be separated. This is done by averaging muonic wave function over the nuclear charge density distributions (to be determined and discussed later). We have then

$$
B_{\Phi K}=\frac{\left\langle\Phi_{\mu}^{2}\right\rangle}{m_{\mu}^{3}} B_{K} .
$$

Thus, while the $B_{\Phi K}$ depends on the bound muon wave function $\Phi$, in the case of the factorization that dependence is separated into the factor $\frac{\left\langle\Phi_{\mu}^{2}\right\rangle}{m_{\mu}^{3}}$. The quantities $B_{K}$ thus are pure nuclear quantities, independent of the muon wave function $\Phi$.

However, as we explain further in Sec. IIC, it is possible to include the bound muon wave function $g_{-1}(r)$ directly, without factorization. We will show later that these two alternatives lead to essentially equivalent resulting capture rates. The final numerical results on the muon capture rate are therefore presented with both the nonfactorization and the traditional way with factorization. Comparison will be presented with the properly normalized $B_{\Phi K}$ :

$$
\widetilde{B}_{K}=\frac{m_{\mu}^{3}}{\left\langle\Phi_{\mu}^{2}\right\rangle} B_{\Phi K} .
$$

\section{Integration with the muon wave function}

In all previous theoretical evaluations of the muon capture rate the factorization in Eq. (24) was used:

$$
\begin{aligned}
\Gamma= & m_{\mu} \frac{\left(G_{\beta} m_{\mu}^{2}\right)^{2}}{2 \pi} \frac{\left\langle\Phi_{\mu}^{2}\right\rangle}{m_{\mu}^{3}} \\
& \times\left(g_{A}^{\text {eff }}\right)^{2}\left(C_{F} \frac{B_{F}}{\left(g_{A}^{\text {eff }}\right)^{2}}+C_{G T} B_{G T}+C_{T} B_{T}\right) .
\end{aligned}
$$

However, if the quatities $B_{K}$ are replaced by $\widetilde{B}_{K}(K=F, G T$, and $T$ ) the capture rate without factorization of muon wave function is obtained.

The squared nuclear matrix elements $B_{F, G T, T}$ in Eq. (26) can be expressed in general as

$$
\begin{aligned}
B_{F} & =\left\langle 0_{i}^{+}\left|\sum_{j k} \tau_{j}^{-} \tau_{k}^{+} \mathcal{F}^{F}\left(\mathbf{r}_{\mathbf{j}}, \mathbf{r}_{\mathbf{k}}\right)\right| 0_{i}^{+}\right\rangle, \\
B_{G T, T} & =\left\langle 0_{i}^{+}\left|\sum_{j k} \tau_{j}^{-} \tau_{k}^{+} \mathcal{F}^{G T, T}\left(\mathbf{r}_{\mathbf{j}}, \mathbf{r}_{\mathbf{k}}, \boldsymbol{\sigma}_{j}, \boldsymbol{\sigma}_{\boldsymbol{k}}\right)\right| 0_{i}^{+}\right\rangle .
\end{aligned}
$$

Let us define the distribution functions $D_{K}\left(r_{1}\right)$, and $D_{K}\left(r_{1}, r_{2}\right)(K=F, G T$, and $T)$ as

$$
\begin{aligned}
D_{F}\left(r_{1}\right)= & \left\langle 0_{i}^{+}\left|\sum_{j k} \tau_{j}^{-} \tau_{k}^{+} \delta\left(r_{1}-r_{j}\right) \mathcal{F}^{F}\left(\mathbf{r}_{\mathbf{j}}, \mathbf{r}_{\mathbf{k}}\right)\right| 0_{i}^{+}\right\rangle \\
D_{G T, T}\left(r_{1}\right)= & \left\langle 0_{i}^{+}\right| \sum_{j k} \tau_{j}^{-} \tau_{k}^{+} \delta\left(r_{1}-r_{j}\right) \\
& \times \mathcal{F}^{G T, T}\left(\mathbf{r}_{\mathbf{j}}, \mathbf{r}_{\mathbf{k}}, \boldsymbol{\sigma}_{j}, \boldsymbol{\sigma}_{\boldsymbol{k}}\right)\left|0_{i}^{+}\right\rangle,
\end{aligned}
$$

as well as

$$
\begin{aligned}
& D_{F}\left(r_{1}, r_{2}\right) \\
& =\left\langle 0_{i}^{+}\left|\sum_{j k} \tau_{j}^{-} \tau_{k}^{+} \delta\left(r_{1}-r_{j}\right) \delta\left(r_{2}-r_{k}\right) \mathcal{F}^{F}\left(\mathbf{r}_{\mathbf{j}}, \mathbf{r}_{\mathbf{k}}\right)\right| 0_{i}^{+}\right\rangle \\
& D_{G T, T}\left(r_{1}, r_{2}\right) \\
& =\left\langle 0_{i}^{+}\right| \sum_{j k} \tau_{j}^{-} \tau_{k}^{+} \delta\left(r_{1}-r_{j}\right) \delta\left(r_{2}-r_{k}\right) \\
& \quad \times \mathcal{F}^{G T, T}\left(\mathbf{r}_{\mathbf{j}}, \mathbf{r}_{\mathbf{k}}, \boldsymbol{\sigma}_{j}, \boldsymbol{\sigma}_{\boldsymbol{k}}\right)\left|0_{i}^{+}\right\rangle
\end{aligned}
$$

Obviously, the $D$ functions are normalized as

$$
\begin{aligned}
B_{K} & =\int_{0}^{\infty} D_{K}\left(r_{1}\right) d r_{1}, \\
B_{K} & =\int_{0}^{\infty} D_{K}\left(r_{1}, r_{2}\right) d r_{1} d r_{2} .
\end{aligned}
$$

Once the $D_{K}\left(r_{1}, r_{2}\right)$ have been calculated one can avoid the factorization of the (averaged) muon wave function and of the sum of squared nuclear matrix elements. The relevant quantity is then

$$
\frac{1}{4 \pi} \frac{1}{m_{\mu}^{3}} \int_{0}^{\infty} g_{-1}\left(r_{1}\right) g_{-1}\left(r_{2}\right) D_{K}\left(r_{1}, r_{2}\right) d r_{1} d r_{2}
$$

instead of $\Phi_{\mu}^{2} / m_{\mu}^{3} B_{K}$ in the Eq. (22). We will show below that the two alternative approaches of treating the bound muon wave function lead to very similar muon capture rates.

\section{Separation of the muon wave function}

For medium and heavy nuclei considered in the present work the relativistic effects on the bound muon are essential, thus the muon wave function is obtained by solving the Dirac equation. In it the nuclear potential is based on the Fermi-type charge distribution, with parameters specified in Table II.

The wave function of the bound $\kappa=-1$ muon is given in Eq. (11). The effect of the nuclear charge distribution on the 
TABLE II. The effective charge $Z_{\text {eff }}$ of Ref. [17] is shown in col. 2 and $Z_{\text {eff }}$ determined in the present work in col. 9. The half-way radius $c_{\mathrm{rms}}$ is in col. 6. The Fermi distribution $\rho(r)=1 /\left\{1+\exp \left[\left(r-c_{\mathrm{rms}}\right) / a\right]\right\}$ is chosen such that the mean square radius $\left\langle r^{2}\right\rangle$ has its experimental value [28] shown in col. 5; the surface thickness $a=0.545 \mathrm{fm}$ is used. The muon wave function $\left\langle\Phi_{\mu}^{2}\right\rangle / m_{\mu}^{3}$ averaged over the nuclear charge distribution $\rho(r)$ is shown in col. 7 using $g_{-1}^{2}(r)+f_{1}^{2}(r)$ and only $g_{-1}^{2}(r)$ in col. $8 . \varepsilon_{b}$ in col 4 is the binding energy of the muon in the $\kappa=-1$ state.

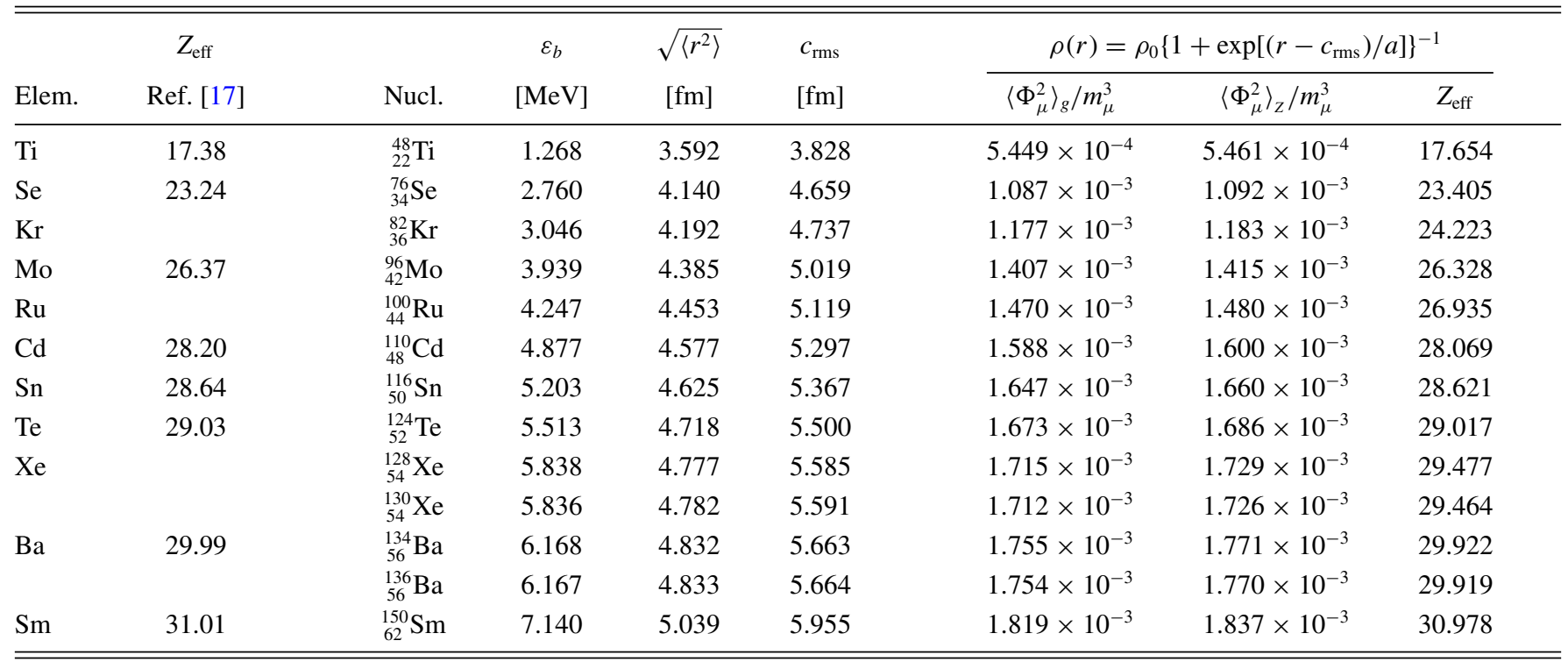

muon, relevant for the muon capture, can be described by the overlap

$$
\left\langle\Phi_{\mu}^{2}\right\rangle_{g}=\int_{0}^{\infty} \frac{g_{-1}^{2}(r)}{4 \pi} \rho(r) r^{2} d r
$$

or, essentially equivalently

$$
\left\langle\Phi_{\mu}^{2}\right\rangle_{Z}=\int_{0}^{\infty} \frac{\left(g_{-1}^{2}(r)+f_{-1}^{2}(r)\right)}{4 \pi} \rho(r) r^{2} d r .
$$

The nuclear charge distribution $\rho(r) \approx 1 /\{1+\exp [(r-$ $\left.\left.\left.c_{\mathrm{rms}}\right) / a\right]\right\}$ is normalized to $Z$, the proton number.

In Fig. 1 we show examples of the radial muon wave functions $g_{-1}(r)$ and $f_{-1}(r)$. Since the small component $f_{-1}(r)$ vanishes at the origin its effect on the muon capture is negligible.

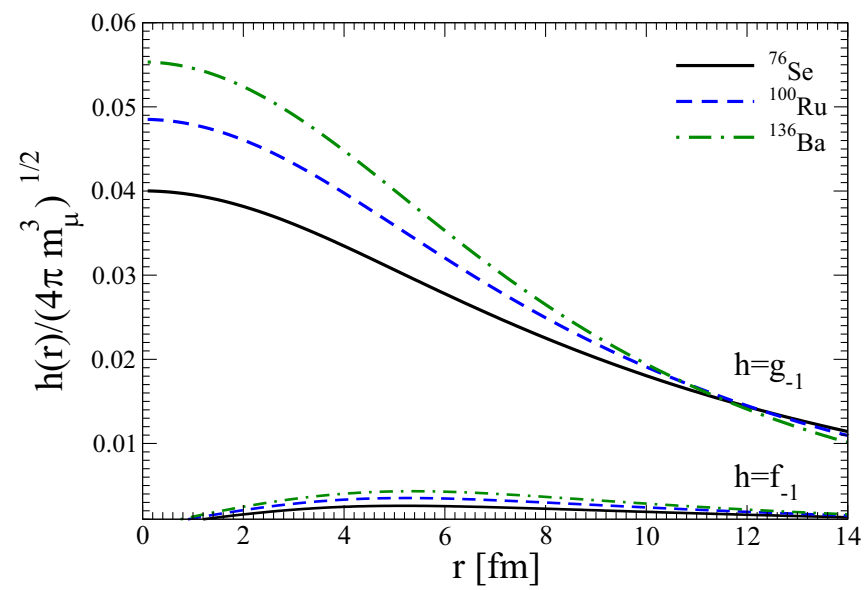

FIG. 1. The radial dependence of the bound muon wave functions $g_{-1}(r)$ and $f_{-1}(r)$ for ${ }^{76} \mathrm{Se},{ }^{100} \mathrm{Ru}$, and ${ }^{136} \mathrm{Ba}$.
Traditionally [27] the quantity $\left\langle\Phi_{\mu}^{2}\right\rangle$ is replaced by the empirical parameter $Z_{\text {eff }}$ using

$$
Z_{\mathrm{eff}}^{4}=\frac{\pi Z}{\alpha^{3}} \frac{\left\langle\Phi_{\mu}^{2}\right\rangle_{Z}}{m_{\mu}^{3}}, \quad \frac{\left\langle\Phi_{\mu}^{2}\right\rangle_{Z}}{m_{\mu}^{3}}=\frac{\alpha^{3}}{\pi Z} Z_{\mathrm{eff}}^{4} .
$$

Using $\left\langle\Phi_{\mu}^{2}\right\rangle_{g}$ instead of $\left\langle\Phi_{\mu}^{2}\right\rangle_{Z}$ makes little difference as seen in Table II. Also, we tested that $Z_{\text {eff }}^{4}$ is insensitive to variations of $c_{\text {rms }}$. By changing it by $1 \%$ changes $Z_{\text {eff }}^{4}$ also by approximately the same amount. Replacing the Fermi distribution by the sharp surface nuclear charge distribution also changes $Z_{\text {eff }}^{4}$ by only a small amount.

\section{E. Evaluation of the matrix elements through a product of two one-body matrix elements}

When considering muon capture on even-even nuclei with the $0^{+}$ground state (all ground states relevant for double- $\beta$ decays are $0^{+}$ground states) we can simplify the evaluation of the quantities $B_{V, A, P}$ (without the factor $\Phi_{\mu}^{2} / m_{\mu}^{3}$ ) in Eq. (14) to find $(I=V, A, P)$

$$
B_{I}=4 \pi \sum_{J_{k}^{\pi}} \frac{E_{v_{k}}^{2}}{m_{\mu}^{2}} B_{I}^{k}\left(J_{k}^{\pi}, p_{v_{k}}\right),
$$

where $p_{v_{k}}=E_{v_{k}}=E_{\mu}+E_{i}-E_{J_{k}^{\pi}}$ and

$$
\begin{gathered}
B_{V, P}^{k}\left(J_{k}^{\pi}, p_{v_{k}}\right)=\left|\sum_{p n}\left\langle n\left\|O_{J}^{V, P}\left(p_{v_{k}}\right)\right\| p\right\rangle T_{p n}\left(J_{k}^{\pi}\right)\right|^{2} \\
B_{A}^{k}\left(J_{k}^{\pi}, p_{v_{k}}\right) \\
=\sum_{L=J, J \pm 1}\left|\sum_{p n}\left\langle n\left\|O_{L J}^{A}\left(p_{v_{k}}\right)\right\| p\right\rangle T_{p n}\left(J_{k}^{\pi}\right)\right|^{2}
\end{gathered}
$$


with

$$
\begin{aligned}
& O_{J M}^{V}\left(p_{v}\right)=i^{J} j_{J}\left(p_{v} r\right) Y_{J M}\left(\Omega_{r}\right), \\
& O_{L J M}^{A}\left(p_{v}\right)=i^{L} j_{L}\left(p_{v} r\right)\left\{Y_{L}\left(\Omega_{r}\right) \otimes \sigma_{1}\right\}_{J M}, \\
& O_{J M}^{P}\left(p_{v}\right)=i^{J-1}\left(\sqrt{\frac{2 J-1}{2 J+1}} j_{J-1}\left(p_{v} r\right)\right. \\
& \quad \times C_{J-1010}^{J 0}\left\{Y_{J-1}\left(\Omega_{r}\right) \otimes \sigma_{1}\right\}_{J M} \\
& \left.\quad-\sqrt{\frac{2 J+3}{2 J+1}} j_{J+1}\left(p_{v} r\right) C_{J+1010}^{J 0}\left\{Y_{J+1}\left(\Omega_{r}\right) \otimes \sigma_{1}\right\}_{J M}\right) .
\end{aligned}
$$

and

$$
\begin{aligned}
T_{p n}\left(J_{k}^{\pi}\right) & =\frac{1}{\sqrt{2 J+1}}\left\langle J_{k}^{\pi}\left\|\left[c_{n}^{+} \tilde{c}_{p}\right]_{J}\right\| 0_{i}^{+}\right\rangle, \\
& =v_{p} u_{n} X_{n p J}^{k}+u_{p} v_{n} Y_{n p J}^{k} .
\end{aligned}
$$

The one-body operators $c_{p}^{+} \tilde{c}_{n}$ (the tilde denotes the timereversed state) appear in the reduced matrix elements. In them, $c_{n}^{+}$creates a neutron, and $c_{p}$ annihilates a proton. Such matrix elements in Eq. (38) depend on the BCS coefficients $u_{\tau}, v_{\tau}$ $(\tau=p, n)$ and on the QRPA vectors $X_{n p J}^{k}$ and $Y_{n p J}^{k}$. The nuclear structure information resides in these quantities.

\section{F. Calculation through two-body matrix elements}

There is an alternative and equivalent way to evaluate the squared matrix elements $B_{F, G T, T} K=$ [Fermi $(F)$, GamowTeller $(G T)$, and tensor $(T)$ ]. It can be expressed as sums over the final states, labeled by their angular momentum and parity $J^{\pi}$ and indices $k$ in the QRPA as follows $(K=F, G T$, and $T)$ :

$$
\begin{aligned}
B_{K}= & \sum_{J_{k}^{\pi}, \mathcal{J}} \sum_{p n p^{\prime} n^{\prime}} \frac{E_{v_{k}}^{2}}{m_{\mu}^{2}} \\
& \times(-1)^{j_{n}+j_{p^{\prime}}+J+\mathcal{J}} \sqrt{2 \mathcal{J}+1}\left\{\begin{array}{lll}
j_{p} & j_{n} & J \\
j_{n^{\prime}} & j_{p^{\prime}} & \mathcal{J}
\end{array}\right\} \\
& \times\left\langle n(1), p^{\prime}(2) ; \mathcal{J}\left\|O_{K}\left(p_{v_{k}}\right)\right\| p(1), n^{\prime}(2) ; \mathcal{J}\right\rangle \\
& \left.\times\left\langle 0_{i}^{+} \| \widetilde{\left[c_{p^{\prime}}^{+} \tilde{c}_{n^{\prime}}\right.}\right]_{J} \| J_{k}^{\pi}\right\rangle\left\langle J_{k}^{\pi}\left\|\left[c_{n}^{+} \tilde{c}_{p}\right]_{J}\right\| 0_{i}^{+}\right\rangle .
\end{aligned}
$$

As in Eq. (35) the reduced matrix elements of the one-body operators $c_{p}^{+} \tilde{c}_{n}$ depend on the BCS coefficients $u_{i}, v_{j}$ and on the QRPA vectors.

The two-body operators $O_{F, G T, T}$ are given by

$$
\left\{\begin{array}{c}
O_{F}\left(p_{v}\right) \\
O_{G T}\left(p_{v}\right) \\
O_{T}\left(p_{v}\right)
\end{array}\right\}=\tau_{1}^{+} \tau_{2}^{-}\left\{\begin{array}{c}
j_{0}\left(p_{v} r_{12}\right) \\
-j_{0}\left(p_{v} r_{12}\right) \sigma_{k l} \\
j_{2}\left(p_{v} r_{12}\right) S_{12}
\end{array}\right\} .
$$

Their matrix elements depend on the relative distance $r_{12}$.

In the above derivation we used

$$
\begin{aligned}
& \int e^{i \mathbf{p}_{v} \cdot \mathbf{r}_{k}} e^{-i \mathbf{p}_{v} \cdot \mathbf{r}_{j}} \frac{d \Omega_{v}}{4 \pi}=j_{0}\left(p_{v} r_{k j}\right), \\
& \int\left(\boldsymbol{\sigma}_{k} \cdot \hat{\mathbf{p}}_{v}\right)\left(\boldsymbol{\sigma}_{j} \cdot \hat{\mathbf{p}}_{v}\right) e^{i \mathbf{p}_{v} \cdot \mathbf{r}_{k}} e^{-i \mathbf{p}_{v} \cdot \mathbf{r}_{j}} \frac{d \Omega_{v}}{4 \pi} \\
& =\frac{1}{3}\left[j_{0}\left(p_{v} r_{k j}\right) \sigma_{k j}-j_{2}\left(p_{v} r_{k j}\right) S_{k j}\left(\hat{r}_{k j}\right)\right]
\end{aligned}
$$

with

$$
\begin{aligned}
\sigma_{k j} & =\boldsymbol{\sigma}_{k} \cdot \boldsymbol{\sigma}_{j} \\
S_{k j}\left(\mathbf{r}_{k j}\right) & =3 \boldsymbol{\sigma}_{k} \cdot \hat{\mathbf{r}}_{k j} \boldsymbol{\sigma}_{j} \cdot \hat{\mathbf{r}}_{k j}-\boldsymbol{\sigma}_{k} \cdot \boldsymbol{\sigma}_{j} .
\end{aligned}
$$

Note that the squared matrix elements $B_{F}, B_{G T}$, and $B_{T}$ are analogous to the matrix elements associated with the secondorder process contributing to electron scattering on nuclei. These matrix elements contain a summation over pairs of nucleons inside the nucleus with relative distance $r_{i j}$.

\section{CHOICE OF INPUT PARAMETERS AND SENSITIVITY OF THE RESULTS}

In this section we discuss the choice of empirical input parameters and the sensitivity of calculated rates to them. The first choice to be made are the nuclear single-particle energies and the corresponding wave functions. The eigenvalues of the Coulomb-corrected Woods-Saxon potential with Bertsch parametrization [29] are used. In order to test the dependence on the single-particle basis we performed our calculation with two choices of single-nucleon basis. The small basis has 11 levels (oscillator shells $N=0-3$ plus the $g_{9 / 2}$ from $N=4$ ) for ${ }^{48} \mathrm{Ti}, 16$ levels (oscillator shells $N=0-4$ plus the $h_{11 / 2}$ from $N=5$ ) for ${ }^{76} \mathrm{Se}$ and ${ }^{82} \mathrm{Kr}, 18$ levels (oscillator shells $N=0-4$ plus the $p_{3 / 2}, f_{7 / 2}$, and $h_{11 / 2}$ from $N=5$ ) for ${ }^{96} \mathrm{Mo}$ and ${ }^{100} \mathrm{Ru}, 21$ levels (oscillator shells $N=0-5$ ) for ${ }^{110} \mathrm{Cd}, 22$ levels (oscillator shells $N=0-5$ plus the $i_{13 / 2}$ from $N=6$ ) for ${ }^{116} \mathrm{Sn},{ }^{124} \mathrm{Te},{ }^{128} \mathrm{Xe},{ }^{130} \mathrm{Xe},{ }^{134} \mathrm{Ba},{ }^{134} \mathrm{Ba}$, and ${ }^{136} \mathrm{Ba}, 23$ levels (oscillator shells $N=0-5$ plus the $g_{9 / 2}$ and $i_{13 / 2}$ from $N=6$ ) for ${ }^{150} \mathrm{Sm}$. All single-particle states in the small basis are bound.

The large model space contains 28 levels (oscillator shells $N=0-6)$ for ${ }^{48} \mathrm{Ti},{ }^{76} \mathrm{Se},{ }^{82} \mathrm{Kr},{ }^{96} \mathrm{Mo},{ }^{100} \mathrm{Ru},{ }^{110} \mathrm{Cd},{ }^{116} \mathrm{Sn}$ and 35 levels (oscillator shells $N=0-7$ without $j_{13 / 2}$ from $N=7$ ) for ${ }^{116} \mathrm{Sn},{ }^{124} \mathrm{Te},{ }^{128} \mathrm{Xe},{ }^{130} \mathrm{Xe},{ }^{134} \mathrm{Ba},{ }^{136} \mathrm{Ba}$, and ${ }^{150} \mathrm{Sm}$. Some of the neutron states in the large basis are quasibound or truly unbound.

Our results suggest that the smaller basis is inadequate since adding additional states changes the capture rate significantly. To test the convergence of the larger single-particle space we checked that subtracting few upper levels makes only small difference.

In QRPA we treat the muon capture as the creation of the correlated proton-hole-neutron-particle states. Experimentally only a fraction of the final states remains bound in the final odd-odd $(Z-1, N+1)$ nucleus, while most final states involve the emission of one or more neutrons. It is therefore clear that highly excited states in the final nucleus are present. Hence, it is important to include in the calculation as many neutron single-particle states above the Fermi level as possible. On the other hand, the quasibound or unbound states included in the large single-particle space in this work do not have the correct asymptotic behavior. It is, therefore, likely that the optimal single-particle space is between the boundaries developed in this work. 


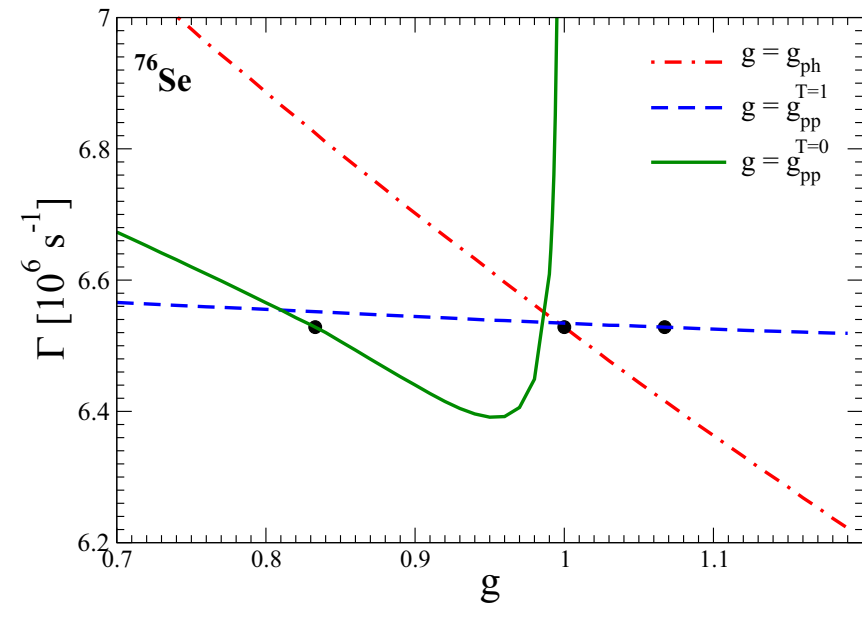

FIG. 2. The muon capture rate of ${ }^{76} \mathrm{Se}$ as function of constants $g_{p h}, g_{p p}^{T=1}$, and $g_{p p}^{T=0}$ used to renormalize, respectively, the particlehole, isovector and isoscalar channels of particle-particle interaction of the nuclear Hamiltonian. The small model space and factorization of muon wave function and nuclear matrix elements is assumed. The positions of the black points indicate the fixed values of these parameters in general calculation of the capture rate, namely $g_{p h}=1.000$, $g_{p p}^{T=1}=1.067$, and $g_{p p}^{T=0}=0.833$.

The residual nuclear interaction enters the QRPA equation of motion. In this work we use the Brueckner $G$-matrix elements [30] calculated with a realistic one-boson-exchange Argonne V18 potential [31].

The pairing interaction has been included in the standard way, i.e., the coupling constant $g_{\text {pair }}$ of the $T=1, J=0$ interaction was slightly renormalized in order to reproduce the experimental pairing gaps. In addition, it is customary in the application of the QRPA method in the evaluation of the $\beta \beta$ nuclear matrix elements to adjust the particle-hole coupling parameter $g_{p h}$ as well as the two isospin components of the particle-particle coupling parameter $g_{p p}^{T}$ (see Refs. [32,33]).

We show an example how the capture rate depends on the renormalized parameters $g_{p h}$ and $g_{p p}^{T}$ in Fig. 2. Over the whole range of realistic $g$ values the capture rate changes by less than $10 \%$, with the exception of the known singularity for $g_{p p}^{T=0}>1$. Our calculation use safely smaller values of $g_{p p}^{T=0}$. For each model space we fixed values of these coupling constants by the condition of the partial restoration of SU(4) symmetry applied for a corresponding double- $\beta$ decay transitions, as described in Ref. [33].

In order to compare the results with and without factorization of the muon wave function, as well as the results with the small and large single-particle model space, we show in Table III the calculated squared nuclear matrix elements $B_{K}$ and $\widetilde{B}_{K}$ [see Eq. (25) for the definition] for the 13 final nuclei participating in the double- $\beta$ decay transitions. Typically, the Gamow-Teller matrix elements are dominant. However, the Fermi and Tensor matrix elements give a non-negligible contributions. Note that the ratio $B_{G T} / B_{F}$ is on average about $2.8 \pm 0.1$, close to the value $B_{G T} / B_{F}=3$ corresponding to the pure $S=0$ state.
TABLE III. The squared matrix elements $B_{K}(K=F, G T$, and $T$ ) for daughter isotopes of the double- $\beta$ decay transitions. $B_{K}$ and $\widetilde{B}_{K}$ correspond to cases with and without factorization of the muon wave function. Small (s) and large (l) single-particle model spaces are considered.

\begin{tabular}{|c|c|c|c|c|c|c|c|}
\hline \multirow[b]{2}{*}{ Nucl. } & \multirow[b]{2}{*}{ m.s. } & \multicolumn{3}{|c|}{$B_{K}$} & \multicolumn{3}{|c|}{$\widetilde{B}_{K}$} \\
\hline & & $\mathrm{F}$ & GT & $\mathrm{T}$ & $\mathrm{F}$ & GT & $\mathrm{T}$ \\
\hline \multirow[t]{2}{*}{${ }^{48} \mathrm{Ti}$} & $\mathrm{s}$ & 2.010 & 5.715 & 0.460 & 1.845 & 5.253 & 0.433 \\
\hline & 1 & 2.379 & 7.308 & 1.239 & 2.230 & 6.829 & 1.269 \\
\hline \multirow[t]{2}{*}{${ }^{76} \mathrm{Se}$} & $\mathrm{s}$ & 3.140 & 8.697 & 1.098 & 2.802 & 7.735 & 1.005 \\
\hline & 1 & 3.620 & 10.37 & 1.948 & 3.336 & 9.473 & 1.931 \\
\hline \multirow[t]{2}{*}{${ }^{82} \mathrm{Kr}$} & $\mathrm{s}$ & 2.938 & 8.356 & 1.107 & 2.614 & 7.385 & 1.001 \\
\hline & 1 & 3.566 & 10.303 & 2.060 & 3.303 & 9.426 & 2.025 \\
\hline \multirow[t]{2}{*}{${ }^{96} \mathrm{Mo}$} & $\mathrm{s}$ & 3.514 & 9.493 & 1.098 & 3.171 & 8.478 & 1.008 \\
\hline & 1 & 4.249 & 12.301 & 2.289 & 3.908 & 11.084 & 2.237 \\
\hline \multirow[t]{2}{*}{${ }^{100} \mathrm{Ru}$} & $\mathrm{s}$ & 3.627 & 9.175 & 1.011 & 3.246 & 8.169 & 0.923 \\
\hline & 1 & 4.485 & 12.765 & 2.290 & 4.091 & 11.405 & 2.229 \\
\hline \multirow[t]{2}{*}{${ }^{110} \mathrm{Cd}$} & $\mathrm{s}$ & 4.028 & 11.593 & 1.845 & 3.629 & 10.175 & 1.669 \\
\hline & 1 & 4.703 & 13.169 & 2.426 & 4.273 & 11.706 & 2.312 \\
\hline \multirow[t]{2}{*}{${ }^{116} \mathrm{Sn}$} & $\mathrm{s}$ & 4.462 & 11.734 & 1.631 & 3.892 & 10.100 & 1.474 \\
\hline & 1 & 4.733 & 12.990 & 2.399 & 4.275 & 11.464 & 2.258 \\
\hline \multirow[t]{2}{*}{${ }^{124} \mathrm{Te}$} & $\mathrm{s}$ & 3.544 & 9.925 & 1.426 & 3.126 & 8.627 & 1.294 \\
\hline & 1 & 3.966 & 11.407 & 2.351 & 3.692 & 10.299 & 2.331 \\
\hline \multirow[t]{2}{*}{${ }^{128} \mathrm{Xe}$} & $\mathrm{s}$ & 3.611 & 10.179 & 1.462 & 3.170 & 8.818 & 1.321 \\
\hline & 1 & 4.193 & 12.084 & 2.455 & 3.876 & 10.864 & 2.414 \\
\hline \multirow[t]{2}{*}{${ }^{130} \mathrm{Xe}$} & $\mathrm{s}$ & 3.277 & 9.452 & 1.415 & 2.906 & 8.229 & 1.282 \\
\hline & 1 & 3.877 & 11.322 & 2.380 & 3.634 & 10.251 & 2.349 \\
\hline \multirow[t]{2}{*}{${ }^{134} \mathrm{Ba}$} & $\mathrm{s}$ & 3.373 & 9.796 & 1.432 & 2.953 & 8.429 & 1.280 \\
\hline & 1 & 4.152 & 12.153 & 2.472 & 3.842 & 10.891 & 2.414 \\
\hline \multirow[t]{2}{*}{${ }^{136} \mathrm{Ba}$} & $\mathrm{s}$ & 3.065 & 9.170 & 1.415 & 2.704 & 7.907 & 1.265 \\
\hline & 1 & 3.866 & 11.505 & 2.449 & 3.617 & 10.357 & 2.394 \\
\hline \multirow[t]{2}{*}{${ }^{150} \mathrm{Sm}$} & $\mathrm{s}$ & 3.575 & 10.057 & 1.561 & 3.247 & 8.964 & 1.427 \\
\hline & 1 & 4.627 & 13.317 & 2.803 & 4.383 & 12.191 & 2.772 \\
\hline
\end{tabular}

The squared matrix elements using the large model space are about 10-20\% larger in comparison with those for the small model space. The capture rate with and without factorization of the muon wave function can be obtained by inserting $B_{K}$ and $\widetilde{B}_{K}$ into Eq. (22), respectively. Thus, a difference of these squared matrix elements quantifies the effect of the factorization treatment. It is not very significant, but is increasing with $Z$ of the nucleus. The entries weakly depend on the $g_{A}^{\text {eff }}$ value. They were evaluated with the $g_{A}^{\text {eff }}$ that reproduces the empirical value of the muon capture rate.

In Table IV we show the contributions of individual $J^{\pi}$ multipoles to the matrix elements and the total capture rate for ${ }^{76} \mathrm{Se}$ and ${ }^{136} \mathrm{Ba}$. The entries were evaluated without the factorization of the muon wave function, using the small and large single-particle model spaces. The present way of choosing the nonrelativistic reduction of the weak Hamiltonian was used. In both cases the $1^{-}, 2^{-}, 1^{+}$, and $2^{+}$multipoles account for $70-80 \%$ of the capture rate.

\section{RESULTS}

As described above we consider several variants when evaluating the muon capture rate. Some of them are preferable, but we comment on the others as well. 
TABLE IV. The multipole decomposition of the matrix elements $\widetilde{B}_{K}(K=V, A, P, F, G T$, and $T)$ [see Eqs. (39) or (35)] and muon capture rate $\Gamma_{\text {pres }}$ for ${ }^{76} \mathrm{Se}$ and ${ }^{136} \mathrm{Ba}$ evaluated in the small (s) and large (1) single-particle model spaces.

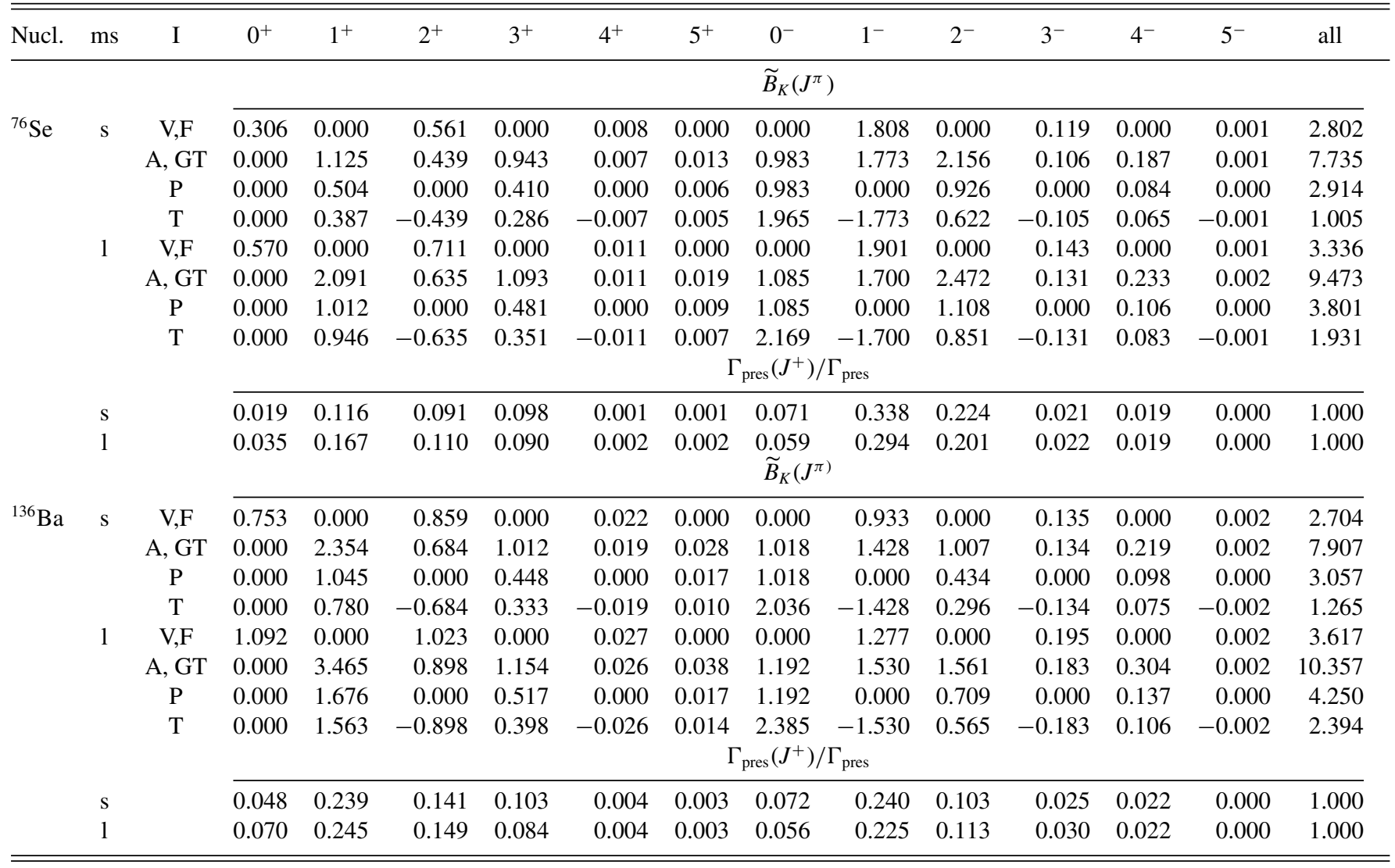

First, two variants, small and large, of the single-particle level set are considered. The larger one seems to be preferable. However, note the issue of the unbound neutron states discussed in Sec. III. As seen in the Table III the calculated capture rates using the small single-particle space are typically $\approx 20 \%$ smaller than those in the large single-particle space. Second, the bound muon wave function can be included in the factorized form, as the $Z_{\text {eff }}^{4}$ factor, or without factorization. Again, we consider the variant without factorization preferable. The corresponding capture rates are $\approx 10 \%$ smaller than those evaluated with factorization. We also verified that the two prescriptions, described in Secs. IIE and IIF lead to the same results. This is an important test of our procedures and codes. Finally, there are two ways of reducing the weak Hamiltonian to its nonrelativistic form. As follows from Table I and the results in this section using the present, and preferable, prescription results in capture rates that are (20-30)\% smaller than those based on the traditional FujiiPrimakoff prescription.

Let us discuss first briefly the energy and multipolarity distributions in the final $(Z-1, N+1)$ odd-odd final nucleus. Note that some, actually most, states in the final nucleus are unbound and lead eventually to the emission of one or more neutrons.

The energy distributions of the final states in muon capture on ${ }^{76} \mathrm{Se}$ and ${ }^{136} \mathrm{Ba}$ are shown in Figs. 3 and 4 . The results with small and larger single-particle spaces are shown. The discrete final states are replaced with the Gaussian peaks of $100 \mathrm{keV}$ width. With the larger single-particle space not only additional higher excitation energy states are populated, but the distribution among the lower-energy states are also noticeably changed. The fraction of bound states below the neutron emission thresholds of $7.33 \mathrm{MeV}$ in ${ }^{76} \mathrm{As}$ is 0.32 for the large single-particle space and 0.36 for the small one. In ${ }^{136} \mathrm{Cs}$ the neutron emission threshold is $6.83 \mathrm{MeV}$, and the

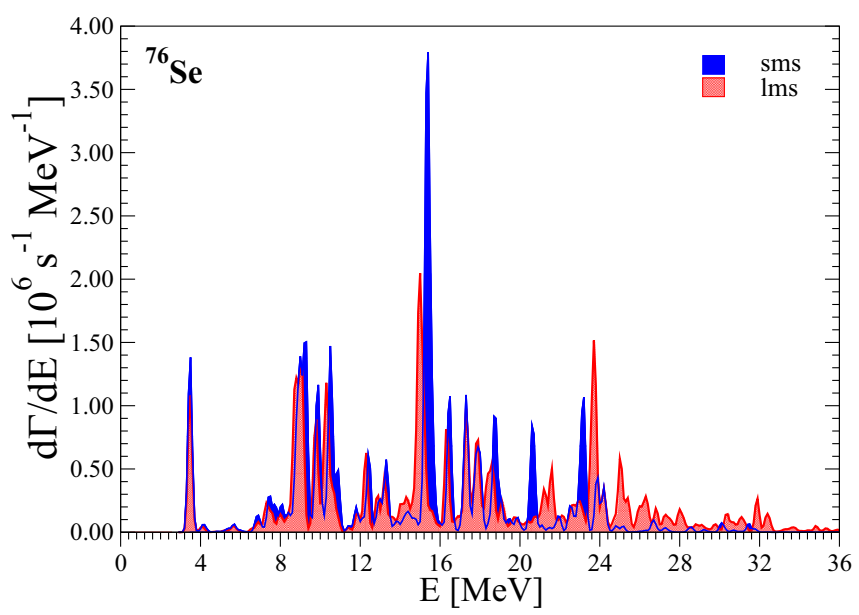

FIG. 3. Energy spectra of muon capture rate on ${ }^{76} \mathrm{Se}$. Results for the small (sms, 22 lev.) and the large (lms, 36 lev.) single nucleon model spaces are presented. 


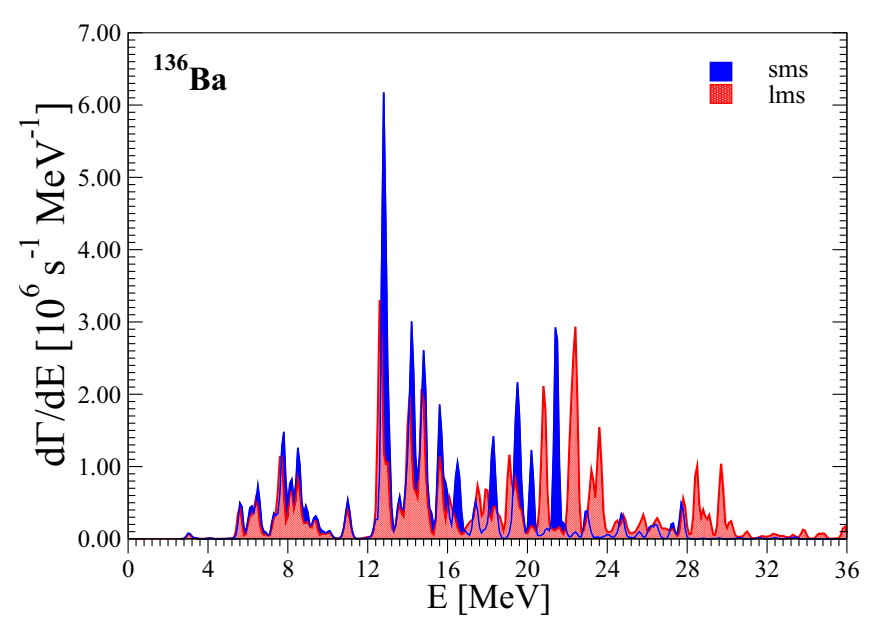

FIG. 4. Energy spectra of muon capture rate on ${ }^{136} \mathrm{Ba}$. Results for the small (sms, 22 lev.) and the large (lms, 36 lev.) single-nucleon model spaces are presented.

corresponding bound state fractions are 0.17 for the large space and 0.21 for the small one.

In Fig. 5 the average excitation energies associated with the largest GT matrix elements are shown for all considered nuclei. The shift between the small and large single-particle spaces, as well as the considerable shift between the evaluation with and without the factorization of the bound muon wave function are clearly visible. However, the pattern of the average excitation energy as a function of the mass number $A$ is very similar in all four variants.

In Figs. 6 and 7 the same energy spectra as in Figs. 3 and 4 are shown, but separated into different multipoles. Only the large single-particle model space is used, hence the scale difference. As is also seen in Table IV the $1^{-}, 2^{-}, 1^{+}$, and $2^{+}$ multipoles dominate, each accounting for roughly comparable contributions.

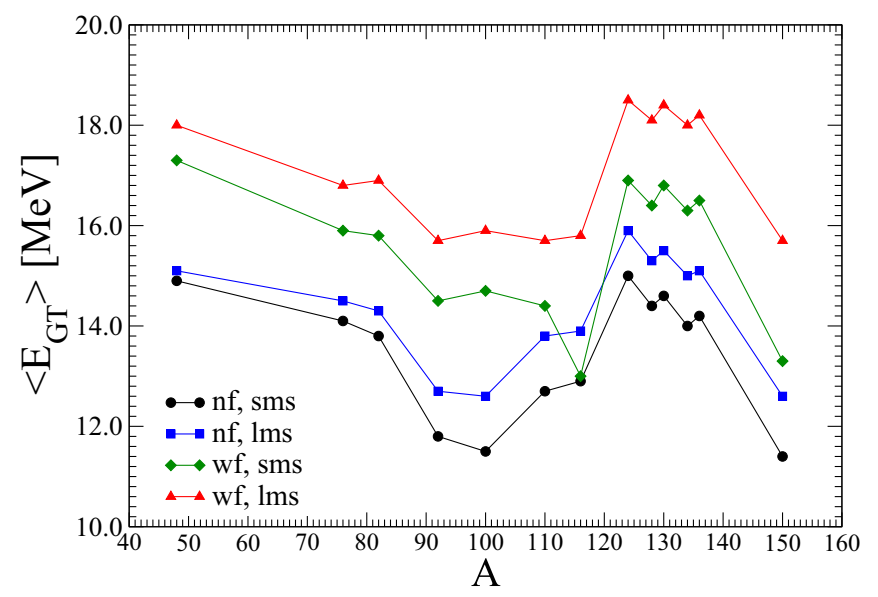

FIG. 5. The average energies of excited state associated with the Gamow-Teller matrix elements $B_{\mathrm{GT}}$ and $\widetilde{B}_{\Phi \mathrm{GT}}$. nf (wf) denotes case without (with) factorization of muon wave function and nuclear matrix element. sms (lms) stands for small (large) model space calculation.

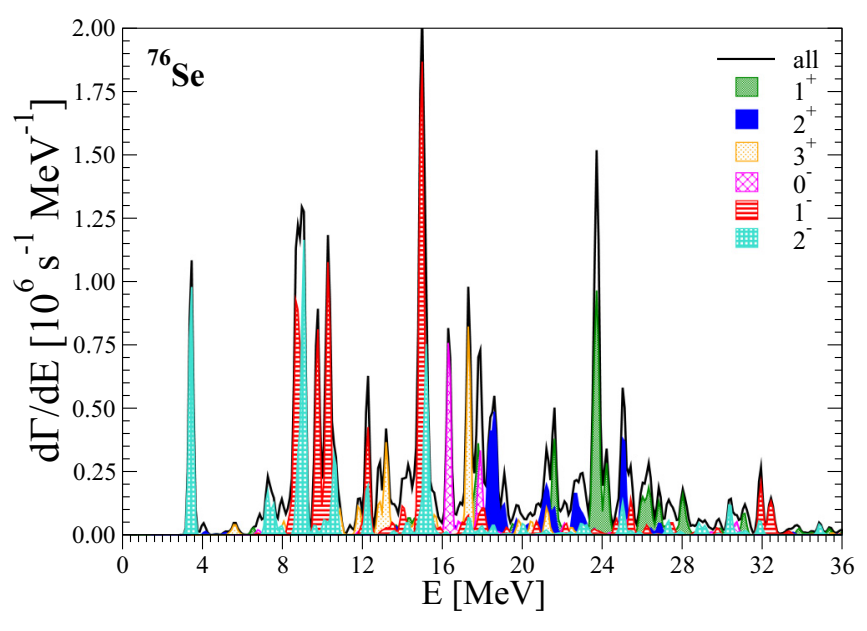

FIG. 6. Multipole contributions to the energy spectra of muon capture rate on ${ }^{76} \mathrm{Se}$ The same notation as in Fig. 3 is assumed. The large single-particle space is considered.

As mentioned in Sec. I, there seems to be a discrepancy between the calculated muon capture rates based on the QRPA method in the older Refs. $[14,16]$ where none, or only mild, quenching of $g_{A}$ was required and more recent Ref. [18] where rather substantial quenching is indicated. To address this discrepancy explicitly using the present method of calculation, we compare in Table V the calculated rates in Ref. [18] and here, with both ways of choosing the constants in the nonrelativistic Hamiltonian. The experimental capture rates $\Gamma_{G P}$, empirically adjusted for the individual isotopes, are also shown. Our results in columns 4 and 5 use, for this purpose only, the same $g_{A}^{\text {eff }}=0.8$ as in Table II of Ref. [18]. One can see that the muon capture rates in Ref. [18] are 2-3 times faster that in our work. At the same time, obviously, the $\Gamma_{Q R P A}$ of Ref. [18] are substantially larger than experiment, thus requiring even smaller $g_{A}^{\text {eff }}$. Our results are smaller than the experiment, thus requiring $g_{A}^{\text {eff }}>0.8$. The origin of the discrepancy is unknown at the present time.

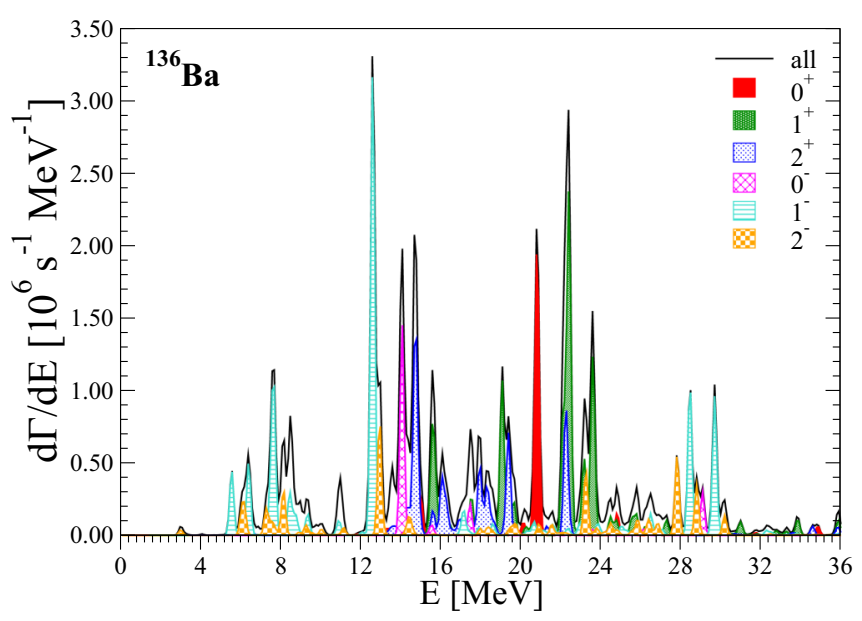

FIG. 7. Multipole contributions to the energy spectra of muon capture rate of ${ }^{136} \mathrm{Ba}$. The same notation as in Fig. 4 is assumed. The large single-particle space is considered. 
TABLE V. Comparison of the experimental muon capture rates $\Gamma_{G P}$, based on the empirical Goulard-Primakoff [5] formula with the rate $\Gamma_{Q R P A}$ calculated in Ref. [18] and those evaluated in this work when using the present $\Gamma_{\text {pres }}$ and the Fujii-Primakoff $\Gamma_{F P}$ parametrizations. Both $\Gamma_{\text {pres }}$ and $\Gamma_{F P}$ were evaluated using the large single-particle model space. All calculations use the same $g_{A}^{\text {eff }}=0.8$, and the rates are in units of $10^{6} / \mathrm{s}$.

\begin{tabular}{lcccc}
\hline \hline nucleus & $\Gamma_{G P}$ & $\Gamma_{Q R P A}$ & $\Gamma_{\text {pres }}$ & $\Gamma_{F P}$ \\
\hline${ }^{76} \mathrm{Se}$ & 7.00 & 16.4 & 3.50 & 4.66 \\
${ }^{82} \mathrm{Kr}$ & 7.22 & 16.5 & 3.76 & 5.00 \\
${ }^{96} \mathrm{Mo}$ & 9.90 & 20.4 & 5.32 & 7.06 \\
${ }^{100} \mathrm{Ru}$ & 11.2 & 16.7 & 5.77 & 7.65 \\
${ }^{116} \mathrm{Sn}$ & 12.7 & 15.7 & 6.61 & 8.73 \\
${ }^{128} \mathrm{Xe}$ & 12.4 & 21.2 & 6.37 & 8.39 \\
${ }^{130} \mathrm{Xe}$ & 11.1 & 23.6 & 5.97 & 7.87 \\
${ }^{136} \mathrm{Ba}$ & 11.1 & 21.1 & 7.61 & 10.0 \\
\hline \hline
\end{tabular}

To see the differences of the results here and in Ref. [18] more clearly we compare in Fig. 8 the results of both works for ${ }^{76} \mathrm{Se}$ and ${ }^{136} \mathrm{Ba}$ used as examples. The differences in both the energy and multipole distributions are quite noticeable. It appears that the present approach leads to somewhat less strength at lower excitation energy and correspondingly more strength at higher energies compared to the results of Ref. [18].

Note, that the experimental data are mostly for elements, not for individual isotopes. Thus, instead of using them directly, we use for comparison with calculations the so-called Goulard-Primakoff empirical formula [5] that describes sufficiently well the muon capture rate for all nuclei with given $A$ and $Z$,

$$
\begin{aligned}
\Gamma_{\mathrm{GP}}^{\mu A}(A, Z)= & Z_{\mathrm{eff}}^{4} G_{1}\left[1+G_{2} \frac{A}{2 Z}-G_{3} \frac{A-2 Z}{2 Z}\right. \\
& \left.-G_{4}\left(\frac{A-Z}{2 A}+\frac{A-2 Z}{8 A Z}\right)\right],
\end{aligned}
$$

where $G_{1}=261, G_{2}=-0.040, G_{3}=-0.26, G_{4}=3.24$ (TRIUMF data fit). While the agreement of the GoulardPrimakoff empirical formula with the few measured capture rates for individual isotopes is not perfect, the discrepancies never exceed $\approx 10 \%$.

Our main results are shown in Fig. 9 where the experimental total capture rates are compared with the calculated rates for $g_{A}^{\text {eff }}=1.27$ and 1.0, and for all final nuclei participating in the double- $\beta$ decay. Clearly, for these results, obtained with the large single-particle model space, the experimental data are bracketed by these two $g_{A}^{\text {eff }}$ values.

More details are shown in Table VI. Here the results with and without muon wave function factorization are shown for both single-particle spaces and for $g_{A}^{\text {eff }}=0.8,1.0$, and 1.27. The experimental data for the natural elements from Ref. [3] and for separated isotopes from Ref. [13] are also shown for comparison.

Finally, in Fig. 10 we display the values of the effective axial current coupling constant $g_{A}^{\text {eff }}$ needed to obtain the empirical total muon capture rate as given by the GoulardPrimakoff formula. It is encouraging to note that for the

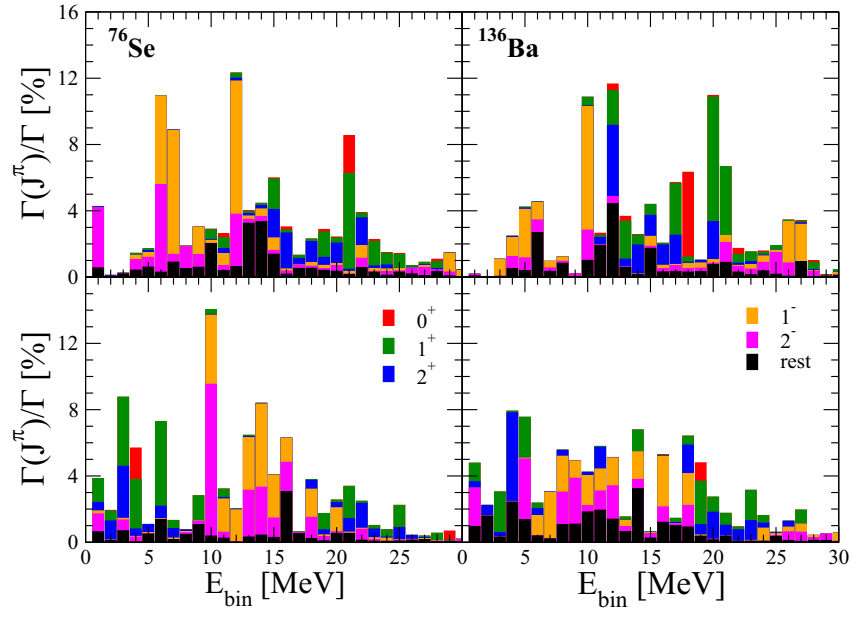

FIG. 8. Multipole and energy distributions of the muon capture rate of ${ }^{76} \mathrm{Se}$ and ${ }^{136} \mathrm{Ba}$ expressed as fractions of the total capture rate. In the top panels are our results, evaluated using the large singleparticle model space. In the bottom panels are the results of Ref. [18]. The energy scale refers to the excitation energy in the final odd-odd nuclei.

preferred variant with the large single-particle space and present prescription of reducing the weak Hamiltonian to its nonrelativistic form (blue line) no quenching at all is required. But given the approximations involved this level of agreement is perhaps somewhat accidental. However, the figure shows clearly that no matter what $g_{A}^{\text {eff }} \geqslant 1.0$ is required to reproduce the experimental muon capture rates.

\section{CONCLUSIONS}

The study of muon capture on nuclei is an important test of the ability of nuclear models to describe this semileptonic weak process. Muon capture is characterized by the relatively

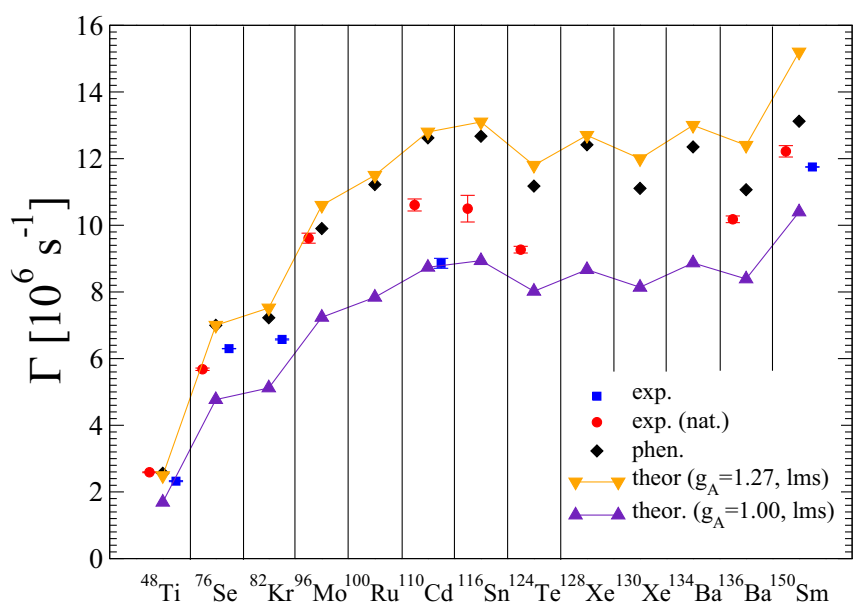

FIG. 9. A comparison of experimental and theoretical total capture rates for the final nuclei participating in the double- $\beta$ decay. Measurements were performed for a for a given isotope in Ref. [13] and for elements with the natural abundance of isotopes in Ref. [3]. Theoretical results were obtained with large model space for $g_{A}=$ 1.00 and 1.27 . 
TABLE VI. The calculated muon capture rates for the final nuclei participating in double- $\beta$ decay. The rates are evaluated for $g_{A}^{\text {eff }}$ values $0.80,1.00$, and 1.27 , as indicated. They are obtained with the present approach with (with fact.) and without (no fact.) factorization of muon wave functions. The small (s) and large (l) single-particle level spaces are considered. $\Gamma_{\text {exp. }}$ is the experimental total capture rate for the stable elements with natural abundance of isotopes [3] and for a given isotope [13]. All values of capture rates are in units $10^{6} s^{-1}$

\begin{tabular}{|c|c|c|c|c|c|c|c|}
\hline \multirow[b]{2}{*}{ nuclide } & \multirow[b]{2}{*}{$\Gamma_{\text {exp. }}$} & \multirow[b]{2}{*}{ isotope } & \multirow[b]{2}{*}{$g_{A}^{\text {eff }}$} & \multicolumn{2}{|c|}{$\begin{array}{c}\quad \Gamma_{\text {pres }} \\
\text { with fact. }\end{array}$} & \multicolumn{2}{|c|}{ no fact. } \\
\hline & & & & $\mathrm{s}$ & 1 & $\mathrm{~s}$ & 1 \\
\hline \multirow[t]{2}{*}{${ }^{\text {nat }} \mathrm{Ti}$} & $2.590 \pm 0.012$ & ${ }^{48} \mathrm{Ti}$ & 0.80 & 1.08 & 1.32 & 0.99 & 1.23 \\
\hline & $2.60 \pm 0.04$ & & 1.00 & 1.47 & 1.81 & 1.35 & 1.69 \\
\hline${ }^{48} \mathrm{Ti}$ & $2.323 \pm 0.015$ & & 1.27 & 2.15 & 2.67 & 1.97 & 2.49 \\
\hline \multirow[t]{2}{*}{${ }^{n a t} \mathrm{Se}$} & $5.681 \pm 0.037$ & ${ }^{76} \mathrm{Se}$ & 0.80 & 3.30 & 3.83 & 2.94 & 3.50 \\
\hline & $5.70 \pm 0.05$ & & 1.00 & 4.47 & 5.22 & 3.98 & 4.77 \\
\hline${ }^{76} \mathrm{Se}$ & $6.300 \pm 0.004$ & & 1.27 & 6.53 & 7.66 & 5.81 & 7.00 \\
\hline \multirow[t]{3}{*}{${ }^{82} \mathrm{Kr}$} & $6.576 \pm 0.017$ & ${ }^{82} \mathrm{Kr}$ & 0.80 & 3.40 & 4.10 & 3.01 & 3.76 \\
\hline & & & 1.00 & 4.61 & 5.60 & 4.08 & 5.12 \\
\hline & & & 1.27 & 6.76 & 8.22 & 5.96 & 7.52 \\
\hline \multirow[t]{3}{*}{${ }^{\text {nat }}$ Mo } & $9.23 \pm 0.07$ & ${ }^{96} \mathrm{Mo}$ & 0.80 & 4.73 & 5.87 & 4.24 & 5.32 \\
\hline & $9.614 \pm 0.15$ & & 1.00 & 6.39 & 8.01 & 5.72 & 7.24 \\
\hline & $9.09 \pm 0.18$ & & 1.27 & 9.30 & 11.8 & 8.32 & 10.6 \\
\hline \multirow[t]{3}{*}{$\mathrm{Ru}$} & & ${ }^{100} \mathrm{Ru}$ & 0.80 & 4.92 & 6.43 & 4.38 & 5.77 \\
\hline & & & 1.00 & 6.60 & 8.74 & 5.88 & 7.84 \\
\hline & & & 1.27 & 9.54 & 12.8 & 8.50 & 11.5 \\
\hline \multirow[t]{2}{*}{${ }^{\text {nat }} \mathrm{Cd}$} & $10.63 \pm 0.11$ & ${ }^{110} \mathrm{Cd}$ & 0.80 & 6.31 & 7.20 & 5.58 & 6.44 \\
\hline & $10.61 \pm 0.18$ & & 1.00 & 8.59 & 9.79 & 7.59 & 8.74 \\
\hline${ }^{116} \mathrm{Cd}$ & $8.86 \pm 0.15$ & & 1.27 & 12.6 & 14.3 & 11.1 & 12.8 \\
\hline \multirow[t]{3}{*}{${ }^{\text {nat }} \mathrm{Sn}$} & $10.70 \pm 0.14$ & ${ }^{116} \mathrm{Sn}$ & 0.80 & 6.90 & 7.43 & 5.96 & 6.61 \\
\hline & $10.44 \pm 0.18$ & & 1.00 & 9.30 & 10.1 & 8.03 & 8.94 \\
\hline & $10.5 \pm 0.4$ & & 1.27 & 13.5 & 14.7 & 11.7 & 13.1 \\
\hline \multirow[t]{3}{*}{${ }^{n a t} \mathrm{Te}$} & $9.27 \pm 0.10$ & ${ }^{124} \mathrm{Te}$ & 0.80 & 5.76 & 6.47 & 5.03 & 5.89 \\
\hline & $9.06 \pm 0.11$ & & 1.00 & 7.83 & 8.83 & 6.83 & 8.02 \\
\hline & & & 1.27 & 11.5 & 13.0 & 9.99 & 11.8 \\
\hline \multirow[t]{6}{*}{$\mathrm{Xe}$} & & ${ }^{128} \mathrm{Xe}$ & 0.80 & 6.05 & 7.03 & 5.26 & 6.37 \\
\hline & & & 1.00 & 8.22 & 9.60 & 7.14 & 8.67 \\
\hline & & & 1.27 & 12.0 & 14.1 & 10.5 & 12.7 \\
\hline & & ${ }^{130} \mathrm{Xe}$ & 0.80 & 5.45 & 6.53 & 4.86 & 5.97 \\
\hline & & & 1.00 & 7.56 & 8.93 & 6.61 & 8.14 \\
\hline & & & 1.27 & 11.1 & 13.2 & 9.70 & 12.0 \\
\hline \multirow[t]{6}{*}{${ }^{\text {nat }} \mathrm{Ba}$} & $10.18 \pm 0.10$ & ${ }^{134} \mathrm{Ba}$ & 0.80 & 5.88 & 7.19 & 5.09 & 6.50 \\
\hline & $9.94 \pm 0.16$ & & 1.00 & 8.03 & 9.84 & 6.93 & 8.87 \\
\hline & & & 1.27 & 11.8 & 14.5 & 10.2 & 13.0 \\
\hline & & ${ }^{136} \mathrm{Ba}$ & 0.80 & 5.43 & 8.11 & 4.72 & 6.14 \\
\hline & & & 1.00 & 7.43 & 10.6 & 6.45 & 8.39 \\
\hline & & & 1.27 & 11.0 & 14.6 & 9.48 & 12.4 \\
\hline${ }^{n a t} \mathrm{Sm}$ & $12.22 \pm 0.17$ & ${ }^{150} \mathrm{Sm}$ & 0.80 & 6.34 & 8.23 & 5.69 & 7.61 \\
\hline \multirow[t]{2}{*}{${ }^{150} \mathrm{Sm}$} & $11.75 \pm 0.07$ & & 1.00 & 8.62 & 11.2 & 7.72 & 10.4 \\
\hline & & & 1.27 & 12.6 & 16.5 & 11.3 & 15.2 \\
\hline
\end{tabular}

large momentum transfer of the order of muon mass, and hence involves many multipolarities and a wide range of excitation energies. The quasiparticle random phase approximation is a method designed to describe collective nuclear effects. Thus, as a test of the method, the total muon capture rate is, we believe, a characteristic that should be considered

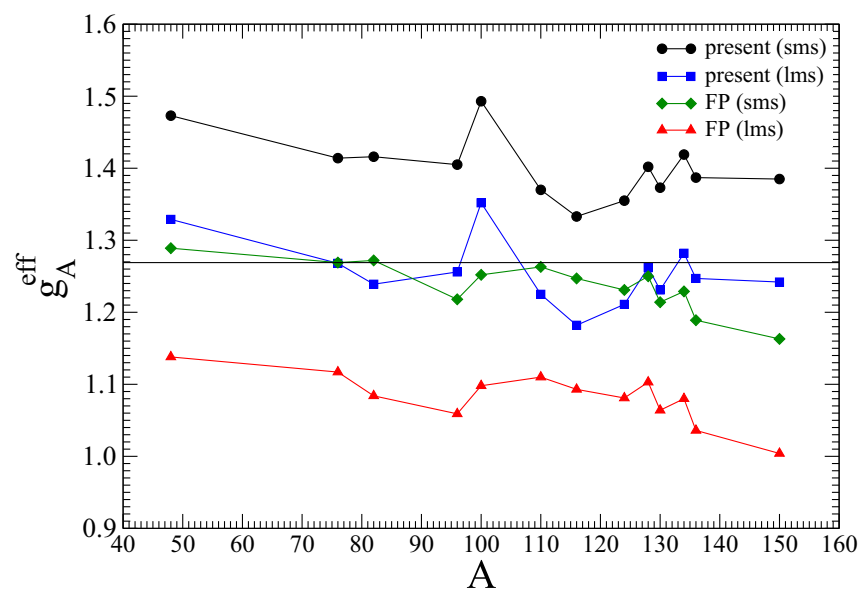

FIG. 10. Effective axial-vector coupling constant $g_{A}^{\text {eff }}$ needed to reproduce the empirical Goulard and Primakoff muon capture rate $\Gamma_{\mathrm{GP}}$. Calculations were performed for the same nuclei as in Fig. 9. Results shown are for the present and Fujii-Primakoff approaches of reducing the weak Hamiltonian to its nonrelativistic form. Both the small (sms) and large (lms) single-particle spaces were used.

first, in preference to the description of the individual final states that each represent only a small fraction of the total capture rate.

In this work we show that the QRPA method is capable to describe the muon capture rate in agreement with experiment in a many nuclei, spanning a large interval of $Z$ and $A$. To reach such a conclusion we used a variety of procedures. Some of them have been used before, some others are new. It is important to note that the resulting calculated capture rates are relatively close to each other, independently of the variant employed. This shows that they are relatively stable. It is also an important test of our procedures, since many of the variants require separate, and seemingly quite different, computer codes.

In particular, our aim is to test whether the idea of the axial current quenching is needed to describe the muon capture. If it would be the case, we would expect that the calculated capture rates would exceed the experimental ones by an approximately constant factor for a large group of nuclei. Our results show that, at least for the QRPA method as described here, this is not the case. We describe the capture rates reasonably well with the standard value of $g_{A}=1.27$. There is no necessity of any quenching. More details, such as the fraction leading to the bound states in the $(Z-1, N+1)$ nucleus, or the branching ratios for the individual bound states, might be also eventually used as additional tests of the model.

\section{ACKNOWLEDGMENTS}

This work was supported by the VEGA Grant Agency of the Slovak Republic under Contract No. 1/0607/20 and by the Ministry of Education, Youth and Sports of the Czech Republic under the INAFYM Grant No. CZ.02.1.01/0.0/0.0/16_019/0000766. The work of P.V. is supported by the Physics Department, California Institute of Technology. 
[1] J. D. Walecka, in Muon Physics II, edited by V. W. Hughes and C. S. Wu (Academic Press, New York, 1975), p. 113.

[2] N. C. Mukhopadhyay, Phys. Rep. C 30, 1 (1977).

[3] D. F. Measday, Phys. Rep. 354, 243 (2001).

[4] M. Eckhause, R. T. Siegel, R. E. Welsh, and T. A. Filipas, Nucl. Phys. 81, 575 (1966).

[5] T. Suzuki, D. F. Measday, and J. P. Roalsvig, Phys. Rev. C 35, 2212 (1987).

[6] B. A. Brown and B. H. Wildenthal, Ann. Rev. Nucl. Part. Sci. 38, 29 (1988).

[7] G. Martinez-Pinedo, A. Poves, E. Caurier, and A. P. Zuker, Phys. Rev. C 53, R2602 (1996).

[8] E. Caurier, F. Nowacki, and A. Poves, Phys. Lett. B 711, 62 (2012).

[9] P. Gysberk et al., Nature Phys. 15, 428 (2019).

[10] J. Barea, J. Kotila, and F. Iachello, Phys. Rev. C 87, 014315 (2013).

[11] V. A. Rodin, A. Faessler, F. Šimkovic, and P. Vogel, Phys. Rev. C 68, 044302 (2003).

[12] F. Šimkovic, A. Faessler, V. Rodin, P. Vogel, and J. Engel, Phys. Rev. C 77, 045503 (2008).

[13] D. Zinatulina, V. Brudanin, V. Egorov, C. Petitjean, M. Shirchenko, J. Suhonen, and I. Yutlandov, Phys. Rev. C 99, 024327 (2019).

[14] N. T. Zinner, K. Langanke, and P. Vogel, Phys. Rev. C 74, 024326 (2006).

[15] E. Kolbe, K. Langanke, and P. Vogel, Phys. Rev. C 62, 055502 (2000).

[16] T. Marketin, N. Paar, T. Niksic, and D. Vretenar, Phys. Rev. C 79, 054323 (2009).
[17] T. Suzuki, S. Chiba, T. Yoshida, K. Takahashi, and H. Umeda, Phys. Rev. C 98, 034613 (2018).

[18] L. Jokiniemi and J. Suhonen, Phys. Rev. C 100, 014619 (2019).

[19] T. Ericson and W. Weise, Pions and Nuclei (Clarendon Press, Oxford, 1988).

[20] E. D. Commins, Weak Interactions (McGraw-Hill, New York, 1973).

[21] V. Bernard, T. R. Hemmert, and Ulf-G. Meißner, Nucl. Phys. A 686, 290 (2001).

[22] M. E. Rose and R. K. Osborn, Phys. Rev. 93, 1315 (1954).

[23] Y. Kohyama and A. Fujii, Prog. Theor. Phys. Suppl. 60, 171 (1976).

[24] A. Lodder and C. C. Jonker, Nucl. Phys. B 2, 383 (1967).

[25] F. Cannata, R. Graves, and H. Uberall, Rivista del Nuovo Cimento 7, 133 (1977).

[26] C. W. Kim and S. L. Mintz, Phys. Rev. C 31, 274 (1985).

[27] K. W. Ford and J. G. Wills, Nucl. Phys. 35, 295 (1962).

[28] I. Angeli and K. P. Marinova, At. Dat. and Nucl. Dat. Tabl. 99, 69 (2013).

[29] G. F. Bertsch, The Practitioner's Shell Model (North Holland, Amsterdam, 1972), p. 206.

[30] H. Müther and A. Polls, Phys. Rev. C 61, 014304 (1999); Prog. Part. Nucl. Phys. 45, 243 (2000).

[31] R. B. Wiringa, V. G. J. Stoks, and R. Schiavilla, Phys. Rev. C 51, 38 (1995).

[32] F. Šimkovic, V. Rodin, A. Faessler, and P. Vogel, Phys. Rev. C 87, 045501 (2013).

[33] F. Šimkovic, A. Smetana, and P. Vogel, Phys. Rev. C 98, 064325 (2018). 